\title{
Storage Life Prediction of Space Relay Based on Elman Neural Network
}

\author{
Wenhua $\mathrm{Li}^{1}$, Lulu Zhou ${ }^{1, \mathrm{a}}$ and Binglong Wang ${ }^{1}$ \\ ${ }^{1}$ Hebei University of Technology, No 8 GuangRong Street HongQiao District Tianjin, China
}

\begin{abstract}
When the linear equation is used to fit the life data for predicting the storage life at room temperature, there is a large error. From this point of view, the parameter degradation is considered and the contact pressure drop parameter is fused according to the comprehensive correlation degree at $60^{\circ} \mathrm{C}, 73^{\circ} \mathrm{C}, 92^{\circ} \mathrm{C}$ and $125^{\circ} \mathrm{C}$. The accelerating factors of four temperature gradients are obtained by using the Arrhenius equation. The parameters and storage life in high temperature state are equivalent to normal temperature. The Elman neural network is used to study and train the fusion parameters, then the storage life of the relay at room temperature is predicted. The prediction error of this method is $2.44 \%$, which is smaller than expected.
\end{abstract}

\section{Introduction}

As a commonly used component in aerospace systems, space relay has the characteristics of 'longterm storage but one use'. The relay will age over time during the storage time, leading to its performance decline even failure, which is directly related to whether the product can be put into use. Therefore, the storage life, as an important technical indicator, is a main parameter to characterize the performance [1].

If there is no test data of the product components during the long-term storage time, under some assumptions, the accelerated life test method is usually used to estimate its life [2]. In some normal circumstances, scholars are used to establish model equations between the acceleration stress and characteristic parameters. Then the storage life under natural conditions can be calculated by fitting the life data. In the paper [3], the aging rate of the product at the storage temperature calculated by the Arrhenius equation is substituted into the fitting equation to predict the storage life at room temperature.

The equation for fitting the life data under each stress does not reflect the process of product change well, and does not take into account the relationship between the stresses. In view of this deficiency, the accelerating factors of four temperature gradients are obtained by the Arrhenius equation according to the fusion value of the contact pressure drop obtained in the previous research. Then the fusion parameters and the storage life at high temperature are equivalent to normal temperature. The Elman neural network is used to study and train the fusion data to predict the storage life of the relay at room temperature.

\footnotetext{
${ }^{\mathrm{a}}$ Corresponding author : 15222200570@163.com
} 


\section{Parameter fusion}

\subsection{Accelerated storage life test}

Oxygen, ultraviolet, vibration, humidity, salt mist, temperature and other environmental factors will cause performance degradation of aerospace relay materials. The accelerated life test is difficult to fully simulate the actual storage profile involved in the environmental factors. In this paper, the relay samples are subjected to an accelerated life test with constant temperature and humidity, and the temperature factor that mainly causes the aging of the sealing materials is selected to be analyzed. The temperature stress is respectively set to 4 stress levels at $60^{\circ} \mathrm{C}, 73^{\circ} \mathrm{C}, 92^{\circ} \mathrm{C}$ and $125^{\circ} \mathrm{C}$.

Two thermostat humidity control boxes are used to simulate two different temperatures. The DC28V voltage is applied to the coils of 25 relays and the DC 6V voltage / $0.1 \mathrm{~A}$ current is applied to the resistive loads. The parameters of all samples under the two temperature levels are monitored regularly at the same time. The monitored parameters in the accelerated life storage test are contact pressure drop, pull-in voltage, release voltage, pull-in time, release time and turn-off voltage. In this paper, the first four parameters of the 100 contacts are recorded continuously for 16 weeks.

\subsection{Parameter fusion}

On the basis of the previous period, the fusion parameter value of the contact pressure drop is obtained by using the comprehensive correlation analysis method to analyze the correlation between the pull-in voltage, the release voltage, the pull-in time parameter and the contact pressure drop.

Assuming that the reference sequence $X_{i}=\left\{x_{i}(k) \mid k=1,2, \ldots, n\right\}$ is the $i$-th standard mode matrix, $Y_{j}=\left\{y_{j}(k) \mid k=1,2, \ldots, n\right\}$ is the $j$-th mode to be examined. Then the similarity coefficient of the two sequences at the $k$-th time is defined as:

$$
\begin{gathered}
r_{i j}=\frac{1}{n} \sum_{k=1}^{n} \frac{\Delta_{\min }+\rho \Delta_{\max }}{\Delta_{i j}(k)+\rho \Delta_{\max }} \\
\Delta_{i j}(k)=\left|X_{i}(k)-Y_{j}(k)\right|
\end{gathered}
$$

Where, $\Delta_{\max }$ is the maximum value of $\Delta_{i j}(k) . \Delta_{\min }$ is the minimum value of $\Delta_{i j}(k) . \rho$ is the resolution coefficient, generally $\rho=0.5$ 。

The relationship between the parameters in the paper is blurred. In the fuzzy clustering system, in order to determine the relationship between the detection data better, a similarity coefficient $\mu_{i j}$ is used to describe the two groups of data [4]. In this paper, the correlation coefficient method is used to calculate $\mu_{i j}$. The formula is as follow:

$$
\begin{gathered}
\mu_{i j}=\frac{\sum_{k=1}^{n}\left|X_{i}(k)-\bar{X}_{i}\right|\left|Y_{j}(k)-\bar{Y}_{j}\right|}{\sqrt{\sum_{k=1}^{n}\left(X_{i}(k)-\bar{X}_{i}\right)^{2} \sum_{k=1}^{n}\left(Y_{j}(k)-\bar{Y}_{j}\right)^{2}}} \\
\bar{X}_{i}=\frac{1}{n} \sum_{k=1}^{n} X_{i}(k), \bar{Y}_{j}=\frac{1}{n} \sum_{k=1}^{n} Y_{j}(k)
\end{gathered}
$$

The degree of relevance reflects the similarity between patterns, which describes the degree of association between the function curves of things over time. Similarity coefficient reflects the similarity between patterns, that is, the difference between the distances of things. The characteristic parameters of each fault mode are usually both random and fuzzy. In order to accurately describe the 
degree of correlation between patterns, the similarity coefficient and the correlation degree are combined to form a comprehensive correlation coefficient in the paper, which can describe the connection between the two series more closely.

The comprehensive correlation coefficient $z_{i j}$ is defined as:

$$
z_{i j}=\alpha r_{i j}+\beta \mu_{i j}
$$

Where, $\alpha$ and $\beta$ are weight coefficients and $\alpha+\beta=1$, reflecting the importance of relevance degree and similarity. The values of $\alpha$ and $\beta$ can also change the influence degree of $\Delta_{\min }$, $\Delta_{\max }$ and $\rho$.

The correlation degree of the three parameters relative to the contact pressure drop at four temperature gradients is analyzed according to the comprehensive correlation analysis. The weight of converted parameter is shown in Table 1. On this basis, multiply the value of each parameter according to the corresponding weight and then sum them, as a result, the fusion contact pressure drop can be obtained. The fusion parameter is used to analyze the overall process of the whole process of change, and is substituted into the Arrhenius equation to convert the temperature.

Table 1. The weight factors of three factors.

\begin{tabular}{|c|c|c|c|c|}
\hline \multirow{2}{*}{ Parameter } & \multicolumn{4}{|c|}{ Comprehensive correlation coefficients } \\
\cline { 2 - 5 } & $60^{\circ} \mathrm{C}$ & $73^{\circ} \mathrm{C}$ & $92^{\circ} \mathrm{C}$ & $125^{\circ} \mathrm{C}$ \\
\hline Pull-up Time[ms] & 0.333 & 0.381 & 0.342 & 0.353 \\
\hline Release Voltage[V] & 0.322 & 0.28 & 0.375 & 0.337 \\
\hline Pull-in Voltage[V] & 0.345 & 0.339 & 0.283 & 0.31 \\
\hline
\end{tabular}

\section{Conversion factor of temperature}

Assuming that the normal working stress level of the product is $S_{0}$, and $S_{k}$ is the $k$-th accelerated stress level. In order to obtain the storage life of the product at room temperature, it is necessary to convert the accelerated stress of the accelerated life test. The Arrhenius model is widely used in the evaluation of accelerated life test when the temperature is used as an accelerated stress [5]. The Arrhenius model linearly unified can be described by the following formula:

$$
\ln R\left(S_{k}\right)=a+b \cdot \psi\left(S_{k}\right)
$$

Where, $R\left(S_{k}\right)$ is the function of reaction rate. $\psi\left(S_{k}\right)$ is the function of the accelerated stress. $a$ and $b$ are estimated constants. The acceleration factor is calculated by (4).

$$
K_{i j}=\exp \left(b \cdot\left[\psi\left(S_{i}\right)-\psi\left(S_{j}\right)\right]\right)
$$

The acceleration factor expression for product in the paper is:

$$
\begin{aligned}
& K_{i j}=\exp \left(b \cdot\left[\frac{1}{T_{j}}-\frac{1}{T_{i}}\right]\right) \\
& T=t+273
\end{aligned}
$$

The value of parameter $b$ is estimated from the fusion parameter.

The Arrhenius equation does not take into account the effect of temperature when calculating the acceleration stress, so the conversion factor is introduced in this paper. The room temperature is selected as $25^{\circ} \mathrm{C}$. The parameters at $125^{\circ} \mathrm{C}$ are converted to $92^{\circ} \mathrm{C}$, and the correlation degree between the converted value and the parameter value at $92^{\circ} \mathrm{C}$ is calculated according to the formula (3). Then the conversion factor of $125^{\circ} \mathrm{C}$ relative to $92^{\circ} \mathrm{C}$ is obtained after multiplying the correlation degree and the accelerating factor resulted from the equation (6). And so on, the gradient conversion factors 
$K_{i j}$ of the high temperature to low temperature are $0.8465,0.9572$ and 0.9301 . The accelerating factor $K$ of $60^{\circ} \mathrm{C}$ relative to $25^{\circ} \mathrm{C}$ is 0.92 according to equation (6). Finally, the fusion data at each temperature are converted to the fusion degradation parameters of the relay at $25^{\circ} \mathrm{C}$.

\section{Prediction of Elman neural network}

\subsection{Elman neural network}

Elman neural network is based on the Jordan network [6]. In contrast to the static feed-forward neural network, in addition to the common input layer, hidden layer and output layer structures, it has a special undertake layer. The undertake layer is similar to the role of the hidden layer, which is the feedback connection within the neural network. The structure of Elman neural network is shown in Figure 1 .

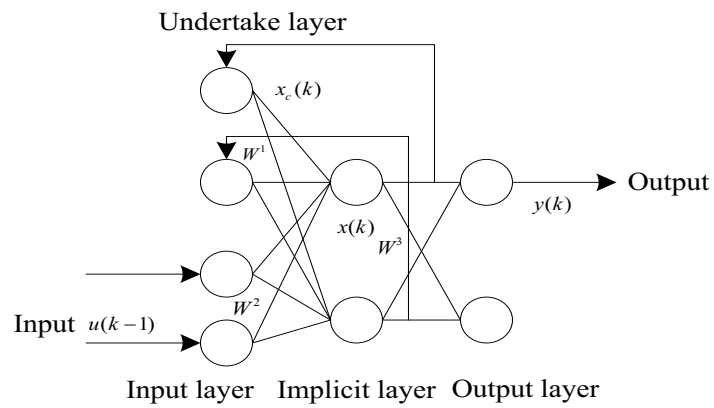

Figure 1. Structure of Elman neural network model.

The nonlinear state space of Elman neural network can be expressed as:

$$
R_{L}(t)=f\left[\sum_{i=1}^{M} W_{1 i j}(t) u_{i}(t)+\sum_{r=1}^{N} W_{2 r j}(t) x_{c r}(t)\right]
$$

Where, $x_{c r}(t)=x_{j}(t-1) . x_{c r}(t)$ is the feedback output of the undertake layer to the hidden layer at time $t$.

The output of the hidden layer to the output layer is:

$$
y_{p}(t)=g\left[\sum_{j=1}^{N} W_{3 j q}(t) x_{j}(t)\right]
$$

Where, $W_{1 i j}(t), W_{2 r j}(t)$ and $W_{3 j q}(t)$ are respectively the weight matrices of the input layer to the hidden layer, the undertake layer to the hidden layer and the hidden layer to the output layer.

The functions of the input layer, undertake layer and output layer are all linear function. In order to speed up the convergence speed of the Elman neural network model, the function of the hidden layer is used modified sigmoid function [7]:

$$
f(x, a, b, c, k)=a+\frac{c}{1+e^{-k(x+b)}}
$$

Where, $a$ is a constant term. $b$ is adjustable bias parameter. $c$ is expanded function gain. The value of the independent variable factor $k$ depends on the input of the neural network

\subsection{Prediction of storage life}

Elman neural network is sensitive to historical data, which increases the ability of network to deal with dynamic information and network stability [8]. The amount of input equivalent data is 1600 . The 
monitoring period of the degraded data is 16 weeks. Therefore, the cycle period of the neural network is 16 , and the network input data is normalized according to Eq. (10).

$$
X_{i}=\frac{x_{i}-x_{\min }}{x_{\max }-x_{\min }}
$$

In order to prevent the extrapolation of the sample data, the training sample is set to 1200 and the test sample is 400. According to the usual method of selecting the number of hidden neurons, the number of hidden neurons is set to 25 and the time of feedback is 4 . The Elman neural network prediction model is established. The training and test results are shown in Figure 2.

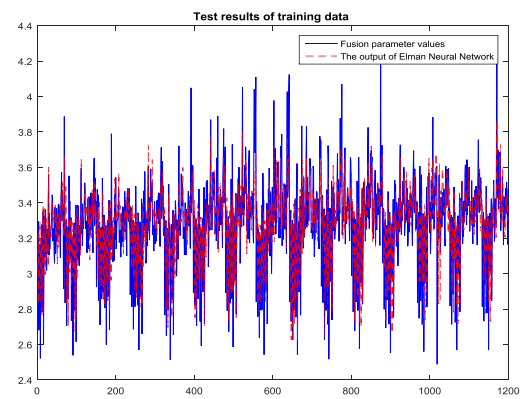

(a) Test results of training data

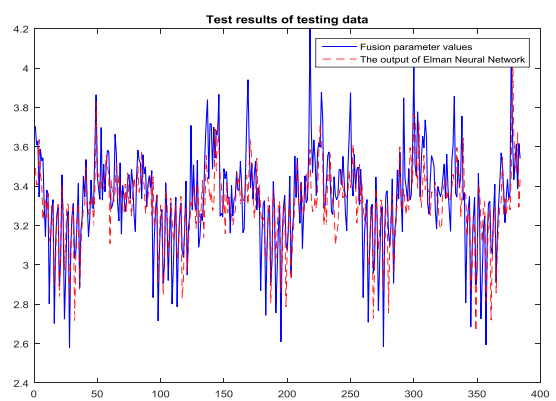

(b) Test results of testing data

Figure 2. The training and test results.

The training mean square error of the Elman neural network $2.44 \%$ and the testing error is $3.25 \%$, which satisfies the expected accuracy. Then the trained network is used to predict the data. When the fused contact pressure drop reaches the equivalent failure threshold of 8.9351, the storage life of the relay at room temperature is 1062 weeks, which is in accordance with the practical engineering application.

\section{Conclusion}

The irrationality of estimating the storage life by linear fitting is taken into account in the paper. The degradation process of the product is studied from the parameter point of view, rather than the simple calculation. The accelerating factors of four temperature gradients are obtained by using the Arrhenius equation, and the parameters and storage life in high temperature state are equivalent to normal temperature. The Elman neural network is used to study and train the fusion parameters, and then the storage life of the relay at room temperature is predicted. The prediction error of this method is $2.44 \%$, which is smaller than expected.

\section{Acknowledgement}

This research was financially supported by Hebei Higher School Science and Technology Research Key Project. (ZD2015051).

\section{References}

1. J. WANG, J. FENG, JY. YANG, et al. Structure\& Environment Engineering, 42, 54-58 (2015)

2. L. JINSUK, P, RONG. IIE Transactions, 42,589-598 (2010)

3. X. Zhou, XJ. Ding, W. Wei. Spacecraft Environment Engineering, 31,287-291 (2014)

4. KT. Chian, FP. Chang, TC. Tsai. International Communications in Heat and Mass Transfer, 33,744-752 (2006) 
5. J. ZHOU, J. YAO, Y. SONG. Journal of Beijing University of Aeronautics and Astronautics, 744750 ( 2015)

6. MI. Jordan. Englewood Cliffs: Erlbaum, 531-546 (1986)

7. R. LIU, GF. FANG. Power System Protection and Control, 40,113-117 (2012)

8. JM. Zhou, ZX. Zhu, YJ. Liu, et al. Journal of Central South University (Science and Technology), 42,3871-3875 (2011) 